\title{
Mathematical model of a flexible asymmetrical rotor for active magnetic bearing reaction wheel
}

\author{
Miroslav Polyakov ${ }^{1,2, * *}$, Anatoliy Lipovtsev ${ }^{1}$, Vladimir Lyanzburg ${ }^{1}$ \\ ${ }^{1}$ Stock Company «Scientific \& Industrial Centre «Polyus», 634050 Tomsk, Russia \\ ${ }^{2}$ National Research Tomsk Polytechnic University, 634050 Tomsk, Russia
}

\begin{abstract}
The paper introduces the mathematical model of rotor for active magnetic bearing reaction/momentum wheels, used as actuator in spacecraft attitude and orbit control system. Developed model is used for estimation of critical speeds and forced oscillation magnitudes with a glance of the rotor modes. Rotor is considered as a two-mass system, consisting of a shaft and a rim, active magnetic bearings are assumed to be a linear elastic springs. The equations of the rotor motion are derived using the Lagrange equation. Developed model is verified by comparing the calculated Campbell diagrams with the results of the finite-element modal analysis, performed in the ANSYS software.
\end{abstract}

\section{Introduction}

Continuous complication of tasks, performed by modern satellites requires the increasing of attitude and orbit control systems operating performance, primarily pointing accuracy and, what is equally important, pointing stability. These parameters are key for high precision optical and astrophysical instruments, used on advanced Earth observation satellites, orbital observatories and telescopes. Nowadays, the need of pointing stability improvement leads to the fact, that the great efforts are applied to solving the microvibration and force-torque disturbances issues. Typically, spacecraft internal disturbances are generated by on-board electromechanical equipment, including reaction wheels (RW) [1-4]. The major RW disturbances sources are ball bearing vibrations, caused by geometrical imperfections, dimensional drift of bearing elements and internal clearances of bearing unit, rotor residual unbalances, determined by the balance quality, control torque ripple, produced by friction torque instabilities [1]. Thus, mainly ball bearings excite the RW micro-disturbances.

Using of active magnetic bearings (AMB) for rotor support is a one of the possible ways to attenuate the RW microvibrations and disturbances. Typically, AMB is a mechatronic system, composed of control object (rotor), actuators (electromagnets), feedback sensors and control system. Significant suppression of the disturbances requires development of the rotor mathematical model, included rotor modes, since they determine the critical speeds and forced oscillations amplitudes inside the gap.

\footnotetext{
* Corresponding author: polyakovmir91@gmail.com
} 


\section{Mathematical model of RW rotor}

RW rotor consists of the rotating parts of electric drive and AMB, mounted on the flywheel. To ensure the optimal mass and inertial characteristics of the rotor (the maximal polar moment of inertia $J_{\mathrm{p}}$ with the minimal mass $M$ ), flywheel comprises of the massive rim, linked to the shaft by a thin lightweight disk (Figure 1a). Results of the RW rotor modal analysis show, that in the case of rigid supports the first natural frequency generally associates with the angular oscillations of the rim and the disk about the lateral axes (Figure 1b). The value of this frequency is rather low and can be spotted in the operating range of rotational speed [5].

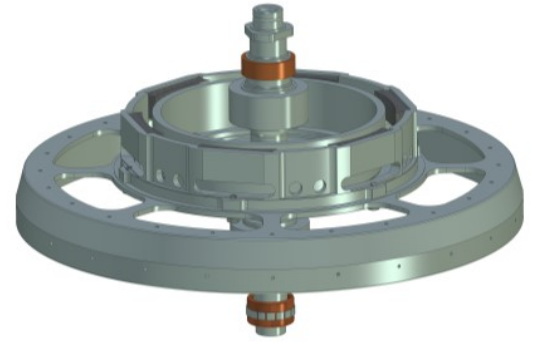

a)

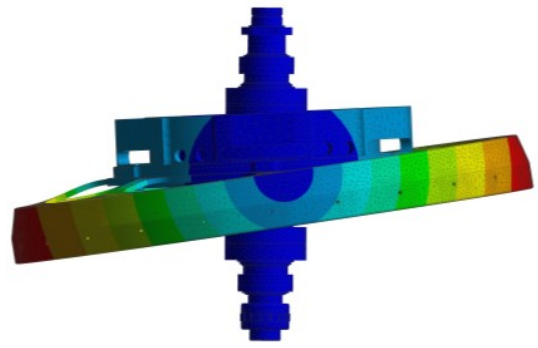

b)

Fig. 1. RW rotor (a) and its first mode (b)

This mode shape allows the RW rotor to be seen as a two-mass system, consisting of the shaft and the rim. The shaft is defined by mass $M_{s}$, polar and equatorial moments of inertia $J_{p . s}, J_{e . s}$, rim is defined by mass $M_{r}$, polar and equatorial moments of inertia $J_{p . r}, J_{e . r}$. There are the following bounds in the system: linear translations and rotation about spin axis are performed by rim and shaft simultaneously (the bound are rigid). The rim rocking relative to the shaft about transversal axes is described by the angular stiffness $R_{d}$.

The following coordinate systems are introduced: $X Y Z$ - frame of the RW; $O X_{s} Y_{s} Z_{s}-$ rotating frame related to the shaft; $O X_{r} Y_{r} Z_{r}$ - rotating frame related to the rim. Oxyz, $O X_{s}^{\prime} Y_{s}^{\prime} Z_{s}^{\prime}, O X_{r}^{\prime} Y_{r}^{\prime} Z_{r}^{\prime}, O X_{s}^{\prime \prime} Y_{s}^{\prime \prime} Z_{s}^{\prime \prime}, O X_{r}^{\prime \prime} Y_{r}^{\prime \prime} Z_{r}^{\prime \prime}$ - intermediate frames, describe the shaft and rim position after linear and angular displacements (Figure 2).

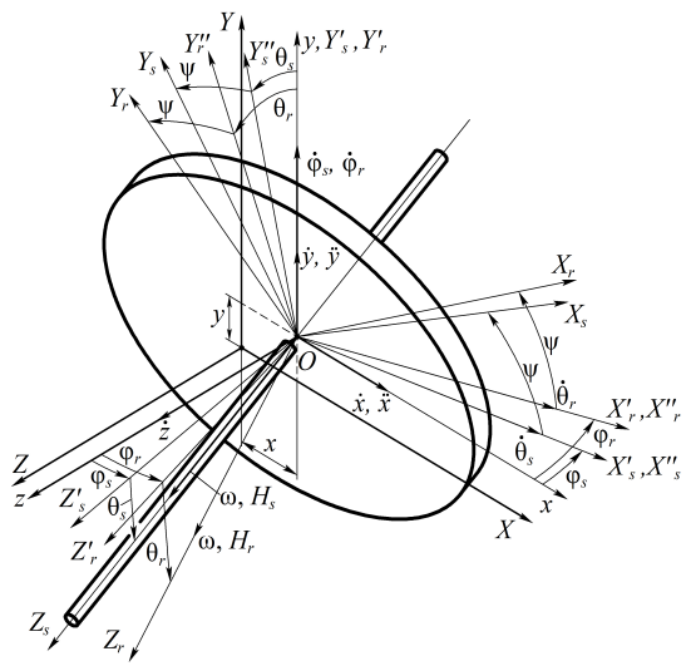

Fig. 2. Model of the flexible RW rotor 
Thereby, subjected to restrains, position of the shaft and rim of the RW rotor is describes by eight generalized coordinates: linear translations of the shaft and rim $x, y, z$, spinning $\psi=\omega t$, shaft and rim rocking about transversal axes with the angles $\varphi_{s}, \theta_{s}$ и $\varphi_{r}, \theta_{r}$ respectively (Figure 2).

Mathematical model of RW rotor is derived by solving the Lagrange equation for every generalized coordinate $q$.

Projection of the angular velocity vectors of the shaft and rim $\omega_{s(r)}$ on the axes of the rotating frames $O X_{s} Y_{s} Z_{s}$ and $O X_{r} Y_{r} Z_{r}$.

$$
\omega_{s(r)}=\left|\begin{array}{c}
\dot{\theta}_{s(r)} \cos \omega t+\dot{\varphi}_{s(r)} \cos \theta_{s(r)} \sin \omega t \\
\dot{\varphi}_{s(r)} \cos \theta_{s(r)} \cos \omega t-\dot{\theta}_{s(r)} \sin \omega t \\
\omega-\dot{\varphi}_{s(r)} \sin \theta_{s(r)}
\end{array}\right| .
$$

Assuming the rocking angles $\varphi_{s}, \theta_{s}, \varphi_{r}, \theta_{r}$ are negligible $\left(\cos \theta_{s(r)} \approx 1, \sin \theta_{s(r)} \approx \theta_{s(r)}\right)$ projection of the angular velocity vectors can be written in the following form

$$
\omega_{s(r)}=\left|\begin{array}{cc}
\dot{\theta}_{s(r)} & \cos \omega t+\dot{\varphi}_{s(r)} \sin \omega t \\
\dot{\varphi}_{s(r)} & \cos \omega t-\dot{\theta}_{s(r)} \sin \omega t \\
\omega-\dot{\varphi}_{s(r)} \theta_{s(r)}
\end{array}\right| .
$$

Axes of rotating frames coincide with the principal axes of inertia, therefore the kinetic energy of the rotor

$$
T=\frac{1}{2}\left[M\left(\dot{x}^{2}+\dot{y}^{2}+\dot{z}^{2}\right)+J_{e s s}\left(\omega_{s X}^{2}+\omega_{s Y}^{2}\right)+J_{p . s} \omega_{s Z}^{2}+J_{e, r}\left(\omega_{r X}^{2}+\omega_{r Y}^{2}\right)+J_{p, r} \omega_{r Z}^{2}\right] .
$$

Finally, the kinetic energy of the rotor is obtained by substituting equation (2) into equation (3)

$$
\begin{aligned}
& T=\frac{1}{2}\left\{M\left(\dot{x}^{2}+\dot{y}^{2}+\dot{z}^{2}\right)+J_{e . s}\right. {\left[\left(\dot{\theta}_{s} \cos \omega t+\dot{\varphi}_{s} \sin \omega t\right)^{2}+\left(\dot{\varphi}_{s} \cos \omega t-\dot{\theta}_{s} \sin \omega t\right)^{2}\right]+} \\
&+J_{p . s}\left(\omega-\dot{\varphi}_{s} \theta_{s}\right)^{2}+J_{e . r}[\left.\left(\dot{\theta}_{r} \cos \omega t+\dot{\varphi}_{r} \sin \omega t\right)^{2}+\left(\dot{\varphi}_{r} \cos \omega t-\dot{\theta}_{r} \sin \omega t\right)^{2}\right]+ \\
&\left.+J_{p . r}\left(\omega-\dot{\varphi}_{r} \theta_{r}\right)^{2}\right\} .
\end{aligned}
$$

The following assumption is made in consideration of potential energy of the system $P$. Since the objective of this stage is consideration and identifications of the dynamic characteristics of single rotor, so it is sufficient to model the radial and axial AMB in the form of ideal elastic and isotropic springs with stiffnesses $C_{A}, C_{B}$ и $C_{Z}$. This assumption, firstly, eliminates the effects of control algorithm and electromagnetic processes in AMB and defines the investigated dynamic behavior of the rotor. Secondly, it gives an opportunity to compare analytical results, obtained by solving the developed model, with the results of modal finite-element analysis. Thus, potential energy $P$ is concentrated in the elastic supports $C_{A}, C_{B}, C_{Z}$ and in the elastic link of the rim and shaft $R_{d}$, so

$$
\begin{aligned}
P=\frac{1}{2}\left[C_{A}\left(x+a \varphi_{s}\right)^{2}+\right. & C_{B}\left(x-b \varphi_{s}\right)^{2}+C_{Z} z^{2}+C_{A}\left(y+a \theta_{s}\right)^{2}+C_{B}\left(y-b \theta_{s}\right)^{2}+ \\
& \left.+R_{d}\left(\varphi_{r}-\varphi_{s}\right)^{2}+R_{d}\left(\theta_{r}-\theta_{s}\right)^{2}\right] .
\end{aligned}
$$

where $a, b$ - distances between center of mass and radial supports, in the case of asymmetrical rotor $a \neq b$. 
Derivatives of Lagrangian for generalized coordinates:

$$
\begin{gathered}
\frac{d}{d t}\left(\frac{\partial L}{\partial \dot{x}}\right)-\frac{\partial L}{\partial x}=M \ddot{x}+C_{A}\left(x+a \varphi_{s}\right)+C_{B}\left(x-b \varphi_{s}\right)=M \ddot{x}+C x+N \varphi_{s} ; \\
\frac{d}{d t}\left(\frac{\partial L}{\partial \dot{y}}\right)-\frac{\partial L}{\partial y}=M \ddot{y}+C_{A}\left(y+a \theta_{s}\right)+C_{B}\left(y-b \theta_{s}\right)=M \ddot{y}+C y+N \theta_{s} ; \\
\frac{d}{d t}\left(\frac{\partial L}{\partial \dot{z}}\right)-\frac{\partial L}{\partial z}=M \ddot{z}+C_{Z} z ; \\
\frac{d}{d t}\left(\frac{\partial L}{\partial \dot{\varphi}_{s}}\right)-\frac{\partial L}{\partial \varphi_{s}}=J_{e . s} \ddot{\varphi}_{s}+J_{p . s} \omega \dot{\theta}_{s}+N x+\left(R_{s}+R_{d}\right) \varphi_{s}-R_{d} \varphi_{r} ; \\
\frac{d}{d t}\left(\frac{\partial L}{\partial \dot{\varphi}_{r}}\right)-\frac{\partial L}{\partial \varphi_{r}}=J_{e . r} \ddot{\varphi}_{r}+J_{p . r} \omega \dot{\theta}_{r}+R_{d} \varphi_{r}-R_{d} \varphi_{s} ; \\
\frac{d}{d t}\left(\frac{\partial L}{\partial \dot{\theta}_{s}}\right)-\frac{\partial L}{\partial \theta_{s}}=J_{e . s} \ddot{\theta}_{s}-J_{p . s} \omega \dot{\varphi}_{s}+N y+\left(R_{s}+R_{d}\right) \theta_{s}-R_{d} \theta_{r} ; \\
\frac{d}{d t}\left(\frac{\partial L}{\partial \dot{\theta}_{r}}\right)-\frac{\partial L}{\partial \theta_{r}}=J_{e . r} \ddot{\theta}_{r}-J_{p . r} \omega \dot{\varphi}_{r}+R_{d} \theta_{r}-R_{d} \theta_{s} ; \\
\frac{d}{d t}\left(\frac{\partial L}{\partial \dot{\psi}}\right)-\frac{\partial L}{\partial \psi}=\left(J_{p . s}+J_{p . r}\right) \ddot{\psi},
\end{gathered}
$$

where $C=C_{A}+C_{B} ; N=C_{A} a+C_{B} b ; R_{s}=C_{A} a^{2}+C_{B} b^{2}$.

Mathematical model of rotor forced oscillations under forces $F_{x}(t), F_{y}(t), F_{z}(t)$ and torques $M_{x}(t), M_{y}(t), M_{z}(t)$ is in the following form:

$$
\left\{\begin{array}{l}
M \ddot{x}+C x+N \varphi_{s}=F_{x}(t) \\
M \ddot{y}+C y+N \theta_{s}=F_{y}(t) \\
M \ddot{z}+C_{Z} z=F_{z}(t) \\
J_{e . s} \ddot{\varphi}_{s}+J_{p . s} \omega \dot{\theta}_{s}+N x+\left(R_{s}+R_{d}\right) \varphi_{s}-R_{d} \varphi_{r}=M_{y}(t) \\
J_{e . r} \ddot{\varphi}_{r}+J_{p . r} \omega \dot{\theta}_{r}+R_{d} \varphi_{r}-R_{d} \varphi_{s}=M_{y}(t) \\
J_{e . s} \ddot{\theta}_{s}-J_{p . s} \omega \dot{\varphi}_{s}+N y+\left(R_{s}+R_{d}\right) \theta_{s}-R_{d} \theta_{r}=M_{x}(t) \\
J_{e . r} \ddot{\theta}_{r}-J_{p . r} \omega \dot{\varphi}_{r}+R_{d} \theta_{r}-R_{d} \theta_{s}=M_{x}(t) \\
\left(J_{p . s}+J_{p . r}\right) \ddot{\psi}=M_{z}(t) .
\end{array}\right.
$$

\section{Results}

Verification of the developed mathematical model of the flexible asymmetrical rotor was carried out by comparing analytical Campbell diagrams, obtained by solving the system (14) with the results of modal finite-element analysis, performed in the ANSYS (Figure 3). 


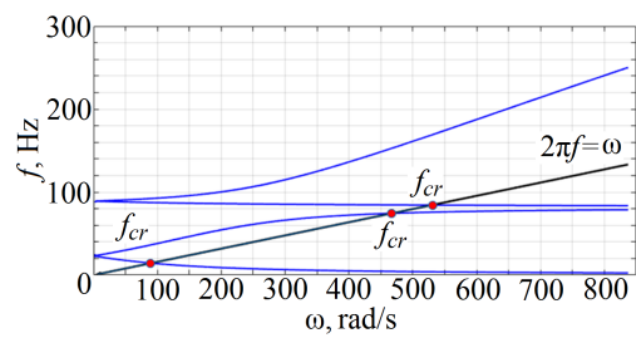

a)

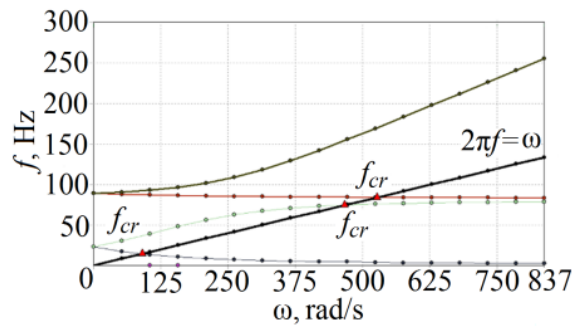

b)

Fig. 3. Campbell Diagrams ( $\mathrm{a}$ - analytical; $\mathrm{b}$ - ANSYS)

Excluding the axial translations $z$ and spinning $\psi=\omega t$, an asymmetric system have three critical speeds (Figure 3), on each speed rotor perform a complex motion, combined of translation and rocking, accompanied by natural oscillations. Analytical critical speeds agree with the results of ANSYS modal analysis, relative error less than $1 \%$ (Table 1).

Table 1. Critical speeds of rotor in the operating range of rotational speed

\begin{tabular}{|c|c|c|c|}
\hline \multirow{2}{*}{ Mode } & \multicolumn{2}{|c|}{ Critical speed, rad/s } & \multirow{2}{*}{ Relative error, \% } \\
\cline { 2 - 3 } & Analytical & ANSYS & \\
\hline Rocking, backward whirl & 91,1 & 90,6 & 0,55 \\
\hline Rocking, forward whirl & 469,8 & 467,1 & 0,58 \\
\hline Radial translation & 533,4 & 529,8 & 0,68 \\
\hline
\end{tabular}

\section{Conclusions}

The developed mathematical model is used for estimation of natural frequencies and critical speeds of flexible rotor of reaction wheel equipped with active magnetic bearings. The relative error of the developed model in comparison with the results of FEM analysis isn't exceed $1 \%$, therefore model can be used for optimization and further investigation of the dynamic behavior of AMB system.

\section{References}

1. M. P. Le, Micro-disturbances in reaction wheels (Technische Universiteit Eindhoven, 2017)

2. K. Komatsu, H. Uchida, Mechanical Engineering Reviews, 1, 00079 (2014)

3. R. Hahn, R. Seiler. 14th European Space Mechanisms \& Tribology Symposium, 415 (2011)

4. G. Smet, G. Richardson, S. McLaren, A. Haslehurst. 15th European Space Mechanisms \& Tribology Symposium, 8 (2013)

5. V. Dmitriev, Y. Britova, MATEC Web of Conferences 48, 01005 (2016) 